\title{
Is air travel safe for patients with cardiac implantable electronic devices?
}

\author{
Aycan Fahri Erkan (D)
}

Department of Cardiology and Aeromedical Centre, Faculty of Medicine, Ufuk University; Ankara-Turkey

\section{ABSTRACT}

Parallel to the rapid expansion of air travel services and advances in medical technology, more passengers with cardiac implantable electronic devices (CIEDs) fly each year. In general, commercial airline flights are considered safe for patients with CIEDs; nevertheless, some specific precautions should be undertaken in some certain circumstances. Apart from the risk of a minor and overlooked pneumothorax early after implantation of a CIED, which may be aggravated due to sudden pressure changes during flight, electromagnetic interference, cosmic radiation and vibration are the other risks a patient with a CIED may encounter during air travel, nevertheless, these are rare and often do not bring about significant clinical consequences.

Keywords: air travel, flight, cardiac implantable electronic devices, pneumothorax, electromagnetic interference, cosmic radiation

\section{Introduction}

Parallel to the rapid expansion of air travel services and advances in medical technology, more passengers with cardiac implantable electronic devices (CIEDs) fly each year. In general, commercial airline flights are considered safe for patients with CIEDs; nevertheless, some specific precautions should be undertaken in some certain circumstances.

\section{Risk associated with pneumothorax early after CIED implantation}

Considering the early period after CIED implantation, it should be borne in mind that pneumothorax may occur as a complication of subclavian vein puncture during pacemaker or implantable cardioverter-defibrillator (ICD) implantation. Although the majority of patients with CIED-implantation-related pneumothorax have a favorable course and are generally managed conservatively, around $0.8 \%$ of them require intervention. Regardless of the necessity of an intervention, air travel should be postponed until 2 weeks after complete radiologic resolution in all cases of CIED-implantation-related pneumothorax. The rationale of this precaution is that gas expansion may occur at low atmospheric pressure at high altitudes, leading to tension pneumothorax and respiratory distress in patients with unresolved pneumothorax (1).

Air travel is generally considered to be safe 1 to 2 days after uncomplicated CIED implantation. However, more prudent physicians recommend postponing air travel for 1 to 2 weeks after a seemingly uncomplicated CIED implantation, advocating that minor pneumothorax may be missed on chest radiographs, and this waiting period may allow for spontaneous healing of these. When the patients are eventually allowed to fly, they should be advised not to carry heavy luggage and not to use the overhead bins lest a lead dislodgement or fracture occurs.

\section{Electromagnetic interference}

Most CIEDs are inherently susceptible to interactions with strong electromagnetic fields, a phenomenon known as electromagnetic interference (EMI). Contemporary devices have special protection against EMl; nevertheless, interference signals can still reach the systems through the leads. Unipolar systems are more vulnerable to EMI than bipolar systems. Most contemporary systems are implanted with bipolar leads, and this has led to a reduction in the incidence of EMI. Hardware and algorithms have been developed and implemented to detect and prevent EMI; and moreover, built-in noise-detection software in modern systems switches to fixed 
rate pacing as a protective measure when EMI is sensed by the system.

Strong magnetic fields may interfere with ICD systems, resulting in inhibition of tachycardia therapies. Such strong fields are created by walk-through security doors in airports; however, most major manufacturers claim that no permanent damage to the CIEDs is anticipated in this setting.

Tiikkaja et al. $(2,3)$ conducted in vitro studies employing explanted or demo implantable cardiac devices to evaluate EMI for low frequency magnetic fields. Implantable devices were subjected to low frequency magnetic fields from $2 \mathrm{~Hz}$ to $1 \mathrm{kHz}$ employing different waveforms including sinusoidal, pulse, ramp, and square waveforms. The information gathered from the findings of these studies can be used to develop standardized test methods for emitters of low frequency magnetic fields. Nevertheless, as Tiikkaja et al. (3) pointed out, the exposure waveform is a crucial parameter in evaluating EMI with active implantable cardiac devices. The waveforms emitted by the walk-through metal detectors (WTMDs) (such as those used in airports) may vary across spatial volume, and this renders the correlation of generalized waveforms to exposure by actual WTMDs complicated.

CIED manufacturers advise that transient and often clinically non-significant interference may occur with WTMDs in airports (4). Although there is a theoretical risk of interference of CIEDs with airport security systems, few cases of interference have been reported. A study from Israel reported no clinically significant interference with walk-through airport security systems in 103 patients with permanent pacemakers who were telemetrically monitored (5). Another study comprised 45 patients with ICDs who were scanned with WTMDs first and then with hand-held metal scanners. ICDs were interrogated after each scan step, and no change in programmed parameters or function was detected (6).

Another study involved 200 patients with permanent pacemakers and 145 patients with ICDs. All the patients were scanned with standard WTMDs and were exposed to the electromagnetic field for at least 20 seconds. No change in programmed parameters, no inappropriate shocks, or inhibition of therapy was observed in this study (7). However, an interrogation of the Food and Drug Administration's manufacturer and user facility device experience (MAUDE) database revealed there were more than 350 incident reports between 2014 and 2016 for certain active medical devices that appear to be related to metal detectors and security systems (8). This evidence points to continuing issues involving EMI via exposure of active medical devices with the airport security systems.

To conclude, although both WTMDs and hand-held detectors in airports seem to be generally safe in patients with CIEDs, it seems prudent to keep the detector wand away from the device pocket, and manual search of patients with ICDs may also be considered as a safety precaution (9). Patients with CIEDs who are planning to travel by plane should be advised that they can be scanned with WTMDs at the airport, and they should pass through them at a normal pace. However, they should also be advised not to spend too much time around the WTMDs to minimize the risk of EMI.

\section{Problems that may be encountered during the flight}

\section{Cosmic radiation}

Most long-range flights operate at a high altitude to reduce fuel costs and avoid turbulence. High-altitude flights are exposed to 100 times more cosmic radiation than that at sea level. Cosmic radiation may cause high energy neutrons to interfere with the integrated random-access memory circuits of the CIEDs. This may theoretically cause single-event upset, especially in the ICD systems, causing the system to switch back to the factory settings. Nevertheless, this is an extremely rare phenomenon, and the ICD switching back to factory settings does not pose an imminent threat to the patient's well-being. Theoretical risks of exposure to cosmic radiation include battery depletion, output inhibition, and failure to detect and treat tachycardia; nevertheless, none of them have been reported during a commercial flight thus far (10).

\section{Vibration}

Pacemakers and ICDs are equipped with motion sensing rate-adaptive sensors. These sensors detect vibrations caused by walking and body motion through piezoelectric crystals. The piezoelectric crystals may also sense vibrations during air travel. Commercial flights generally do not cause significant vibration; however, some vibration may occur during take-off, landing, and turbulence, leading to a slight increase in output rate. This condition does not result in any significant clinical consequences. Helicopter flights, on the other hand, are associated with significant vibration, which may lead to a marked increase in output rate of the pacemaker, causing clinical symptoms. Thus, it is advised to turn off or decrease the rate adaptive function of the pacemaker before a helicopter flight (11). If no programming device is available, in such a situation, a magnet may be placed over the pacemaker to cancel the rate-adaptive function and switch to fixed-rate pacing.

Conflict of interest: None declared.

\section{References}

1. Smith D, Toff W, Joy M, Dowdall N, Johnston R, Clark L, et al. Fitness to fly for passengers with cardiovascular disease. Heart 2010; 96 Suppl 2: ii1-16. [Crossref]

2. Tiikkaja $M$, Alanko $T$, Lindholm $H$, Hietanen $M$, Hartikainen $\mathrm{J}$ Toivonen L. Experimental study on malfunction of pacemakers due to exposure to different external magnetic fields. J Interv Card Electrophysiol 2012; 34: 19-27. [Crossref]

3. Tiikkaja $\mathrm{M}$, Alanko $\mathrm{T}$, Lindholm $\mathrm{H}$, Hietanen $\mathrm{M}$, Toivonen $\mathrm{L}$, Hartikainen J. Interference of low frequency magnetic fields with implantable cardioverter-defibrillators. Scand Cardiovasc J 2012; 46: 308-14. [Crossref]

4. Medtronic Corporation. Guidelines: traveling and security systems. Available from: URL: http://www.medtronic.com

5. Copperman Y, Zarfati D, Laniado S. The effect of metal detector gates on implanted permanent pacemakers. Pacing Clin Electrophysiol 1988; 11: 1386-7. [Crossref] 
6. Kattenbeck K, Achtelik M, Weissmueller P, Trappe HJ. Interactions between implantable cardioverter defibrillators and metal detectors at airports: present or not? Europace Suppl 2000; 1: D101.

7. Kolb C, Schmieder S, Lehmann G, Zrenner B, Karch MR, Plewan A, et al. Do airport metal detectors interfere with implantable pacemakers or cardioverter-defibrillators? J Am Coll Cardiol 2003; 41: 2054-9. [Crossref]

8. Manufacturer and user facility device experience database. Available from: URL: https://www.accessdata.fda.gov/scripts/cdrh/ cfdocs/cfmaude/search.cfm.
9. Boivin W, Coletta J, Kerr L. Characterization of the magnetic fields around walk-through and hand-held metal detectors. Health Phys 2003; 84: 582-93. [Crossref]

10. Ferrick AM, Bernstein N, Aizer A, Chinitz L. Cosmic radiation induced software electrical resets in ICDs during air travel. Heart Rhythm 2008; 5: 1201-3. [Crossref]

11. Flaherty G, Freitas SD. A Heart for Travel: Travel Health Considerations for Patients with Heart Disease and Cardiac Devices. Ir Med J 2016; 109: 486. 